\title{
Applyingthe Feedback Principle for Solving the Equations of Transformers and Electrical Machines
}

ZHULOVYAN Vladimir V. (Novosibirsk State Technical University) - Professor, Dr. Sci. (Eng.)

\begin{abstract}
Anapproach is suggested according to which the primary winding resistance istakeninto account using the well-known feed back principle. The search procedure involves two stages. In the first stage, a voltage source is applied behind the resistance. Then, if flux linkages of the loops are taken as independent variables instead of currents, the order of the equation system to be solved is decreased by unity, a circumstance due to which both the search and the form of sought solutions become essentially simpler. Atthesecondstage, a mathematically rigorous correlation between the conditional and actual voltage across the terminals is found using atechnique known as the feed back principle, as a result of which the preliminarily found quantities are presented as functions of the actual voltage across the terminals. Examples demonstrating the validity and effectiveness of the proposed approach are given as applied to a number of problems concerned with studying steady-state and transient modes of electrical machines and transformers.
\end{abstract}

$\mathrm{K}$ e y w o r d s: electrical machines, transformers, primary winding, resistance, feedback, equation system's order

\section{REFERENCES}

1. White D., Woodson G. Electromechanical energy conversion. Moscow, Publ. «Energiya», 1964, 526 p.

2. Kazovsky E.Y. Transient processes in electrical machines of alternating current. Publ. USSR Academy of Sciences, 1962, 624 p.

3. Kovács K., Rácz I. Transients in machines AC. Moscow, Gosenergoizdat,1963, 738 p.
4. Zhulovyan V. Electrical machines: Electromechanical energy conversion. - Moscow, Publ. «Yurayt», 2017, 427 p.

5. Gurin J.S., Kuznetsov B.I. Series of electric machines designing. Moscow, Publ. «Energiya», 1978, 480 p.

6. Richter R. The electric machine. Vol. 4. The induction machine. Moscow; Leningrad, Gosenergoizdat, 1939, 472 p.

[13.09.2017]

Электричество, 2017, № 12, с. 36-42

DOI:10.24160/0013-5380-2017-12-36-42

\section{Диагностика линейных электрических цепей с косвенной компенсацией подсхем при многократном эксперименте ${ }^{1}$}

\author{
ГОРШКОВ К.С., КУРГАНОВ С.А., ФИЛАРЕТОВ В.В.
}

\begin{abstract}
Задача диагностики произвольной подсхемы в составе линейной электрической цепи сведена к задаче анализа схем, полученных по результатам многократного эксперимента на основе теоремы о косвенной компенсации. Предложенная схемно-алгебрачческая формула в отличие от узловой блочно-матричной позволяет выполнить диагностику в произвольном координатном базисе и получить непосредственно любые параметры исследуемой подсхемы. Для реализации формулы можно использовать известные алгоритмы и программы символьного или численного анализа электрических иепей.
\end{abstract}

К л ю ч е в ы е с л о в а: электрические цепи, диагностика, многократный эксперимент, компенсационный подход, фиксаторы напряжения и тока

В задаче диагностики электрических цепей требуется по результатам эксперимента определить неизвестные параметры элементов [1-7]. Задача решается на основе однократного эксперимента и называется базисной, если число точек, доступных для измерения тока или напряжения, не меньше числа неизвестных параметров [1]. В противном случае требуется проведение многократного (тестового) эксперимента [2], по результатам которого формируются и решаются уравнения в узловом [3] или расширенном узловом базисе [4]. Если искомыми являются параметры элементов, сосредоточенные в одной части схемы, то для нахождения параметров такой подсхемы применяется узловой блочно-матричный метод диагностики [5].

Этот метод предназначен для диагностики автономных (с независимыми источниками) и неавтономных подсхем в цепях, в которых доступных из- 
мерительных точек меньше числа элементов с неизвестными параметрами, но не меньше числа $n$ независимых полюсов исследуемой подсхемы. При этом число экспериментов для диагностики автономной и неавтономной подсхем должно быть не менее $(n+1)$ и $n$ соответственно. Схемы экспериментов отличаются состоянием доступных полюсов - разомкнуты или соединены накоротко (через сопротивление) с общим полюсом или между собой. При разомкнутых полюсах измеряется напряжение, а при замкнутых - ток. По результатам экспериментов формируется блочно-матричная система узловых уравнений, из которой находится матрица узловых проводимостей и искомые проводимости элементов исследуемой подсхемы.

В то же время узловой блочно-матричный метод ограничен элементным составом - допускаются только элементы в базисе проводимостей, что не позволяет диагностировать схемы в произвольном элементном базисе и получить непосредственно не только $Y$, но и $Z$, и гибридные параметры.

Не имеет ограничений на элементный состав компенсационный подход [6], который разработан для диагностики электрических цепей на основе однократного эксперимента и позволяет использовать произвольные двухполюсные элементы и управляемые источники. Подход основан на теореме о косвенной компенсации [6] и методе схемных определителей [8] и состоит в построении эквивалентной схемы с компенсированными элементами (СКЭ) путем замены элементов с неизвестными параметрами нораторами при одновременной установке измеренных переменных с помощью фиксаторов тока и напряжения, содержаших независимые источники и нуллаторы.

Для построения компактных выражений искомых параметров все независимые источники в схеме преобразуются в источники, управляемые нуллатором, совокупность которых называется многомерным управляемым источником [9]. По результатам анализа СКЭ формируется схемно-алгебраическая формула (САФ) для искомого параметра в виде отношения схемных определителей. Так, для искомого сопротивления САФ имеет вид [6]:

$$
Z=-\Delta_{Z} / \Delta^{Z}
$$

где $\Delta_{Z}, \Delta^{Z}-$ определители схем, полученных из СКЭ путем замены проводником и удаления соответственно сопротивления $Z$. Формулы вида (1) реализованы в программе символьного анализа и диагностики CIRSYM [10], которая имеет online-сервис на сайте http://intersyn.net/cirsym. html.
Для диагностики на основе многократного эксперимента в [7] доказана теорема о компенсации подсхемы и рассмотрена возможность применения компенсационного подхода к диагностике неавтономных подсхем. В настоящей статье на основе компенсационного подхода предлагается схемно-алгебраическая формула для определения параметров произвольных, в том числе автономных, подсхем.

Уравнение автономной подсхемы. Автономная подсхема $A_{?}$ с $n$ независимыми полюсами, недоступными для измерений, соединяется с подсхемой $\hat{A}$, имеющей известные параметры и $n$ доступных полюсов 1', 2', $\ldots, n^{\prime}$ (рис. 1,a). Эти полюсы можно соединять с общим полюсом и между собой накоротко через известное сопротивление или тестовый источник тока. Требуется определить $Y, Z$ или гибридные параметры автономной подсхемы $A_{\text {? }}$.

Матричная формула автономной подсхемы с $n$ полюсами имеет вид

$$
W=\Pi X+T,
$$

где $W, X$ - векторы комплексных откликов и воздействий размерностью $n$, каждый из которых может содержать как токи, так и напряжения узлов (сторон) подсхемы в зависимости от заданного типа матрицы параметров П, которая имеет размерность $n \times n ; T-$ вектор размерностью $n$ напряжений холостого хода и токов короткого замыкания на полюсах (сторонах) подсхемы.

Если известны воздействия и отклики $(n+1)$-го режима подсхемы $A_{\text {? }}$, то, объединяя их на основе уравнения (2), можно исключить неизвестный вектор $T$ :

$$
W^{\delta}=\Pi X^{\delta}
$$

где $X^{\delta}=\left(x_{j k}^{\delta}\right)$, и $W^{\delta}=\left(w_{j k}^{\delta}\right)-$ матрицы размерностью $n \times n$ разностей воздействий и разностей откликов: $x_{j k}^{\delta}=x_{j k}-x_{f 0} ; w_{j k}^{\delta}=w_{j k}-w_{j 0} ; j, k=1, \ldots, n-$ номер полюса и эксперимента соответственно; $x_{j 0}$, $w_{j 0}-$ воздействие и отклик на $j$-м полюсе при эксперименте с номером $k=0$.

Из (3) можно найти матрицу параметров подсхемы $A_{\text {? }}$. При этом матрицы $X^{\delta}$ и $W^{\delta}$ предлагается определять путем расчета схем, построенных с учетом теоремы о косвенной компенсации на основе измерений на узлах подсхемы $\hat{A}$.

Расчетные схемы на основе многократного эксперимента и теоремы о косвенной компенсации. Для определенности считаем, что в $k$-м $(k=1,2, \ldots, n)$ эксперименте полюс с номером $j^{\prime}=k$ содинен с общим полюсом (рис. 1,a), а эксперимент с номером 
$k=0$ проводится при разомкнутых полюсах подсхемы $\hat{A}$. На разомкнутых полюсах измеряются комплексные напряжения $\underline{U}_{V 1}, \underline{U}_{V 2}, \ldots, \underline{U}_{V n}$, а в проводнике, замыкающем полюс, - ток $\underline{I} A j$. Напряжение в СКЭ на рис. 1,б устанавливается с помощью фиксаторов напряжения, ЭДС которых $\underline{E}_{V 1}=\underline{U}_{V 1}$, $\underline{E}_{V 2}=\underline{U}_{V 2}, \ldots, \underline{E}_{V n}=\underline{U}_{V n}$, а измеренный ток - с помощью фиксатора тока с источником $\underline{J}_{A j}=\underline{I}_{A j}$.

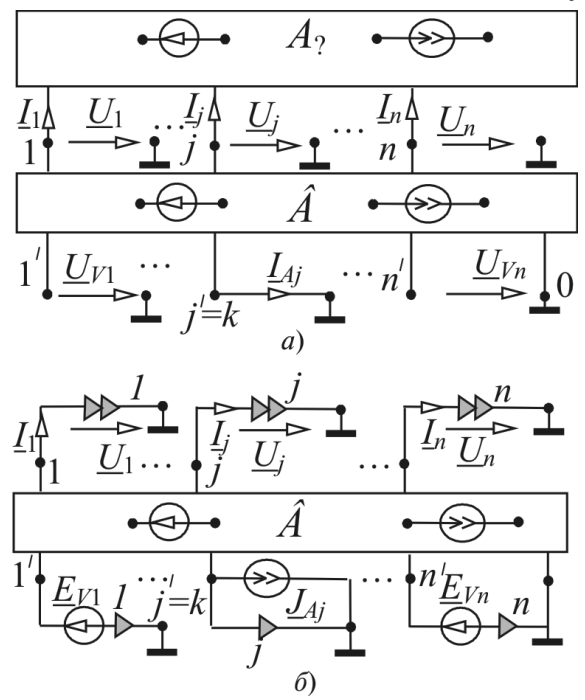

Рис. 1. Схема эксперимента с номером $k(a)$ и эквивалентная СКЭ (б); заштрихованными одинарной и двойной стрелками обозначены нуллатор и норатор соответственно

Схемно-алгебраическая формула для параметров произвольной подсхемы. По схеме на рис. 1,б запишем схемно-алгебраическую формулу (САФ) для напряжения полюса $j$ исследуемой подсхемы при эксперименте $k$ :

вующего элемента в схеме на рис. 1,б проводником (или замыкание стороны полюса с общим полюсом) и удаление элемента из схемы (или размыкание полюса) соответственно. Символ $\Delta_{j}(k)$ означает, что в схеме числителя полюс $j$ соединен проводником с общим полюсом. В (5) учтено изменение знака определителя на противоположный из-за взаимной замены номеров у нуллаторов $j$ и $n+1$ при раскрытии определителя. При построении формул вида (4) и (5) для эксперимента с номером $k=0$ все полюсы в схеме на рис. $1, a$ разомкнуты.

В схеме числителя для тока $I_{j}(k)$ в отличие от схемы числителя для напряжения полюс $j$ подсхемы будет разомкнутым, поскольку здесь норатор и нуллатор соединены последовательно. Знак перед выражением - положительный, поскольку выполняются две операции, изменяющие знак определителя - уже упомянутая взаимная замена номеров у нуллаторов, и удаление последовательного согласного соединения норатора и нуллатора. Таким образом, формула для тока произвольного полюса $j$ исследуемой подсхемы при эксперименте $k$ имеет вид

$$
I_{j}(k)=-\Delta^{j}(k) / \Delta_{E}^{J}
$$

После умножения обеих частей уравнения (3) на их общий знаменатель $\Delta_{E}^{J}$ получаем

$$
N_{W}^{\delta}=\Pi N_{X}^{\delta}
$$

где $N_{X}^{\delta}=\left(v_{j k}^{\delta}\right), N_{W}^{\delta}=\left(\mu_{j k}^{\delta}\right)-$ матрицы размерности $n \times n$ разностей числителей воздействий и разностей числителей откликов подсхемы.

(4)

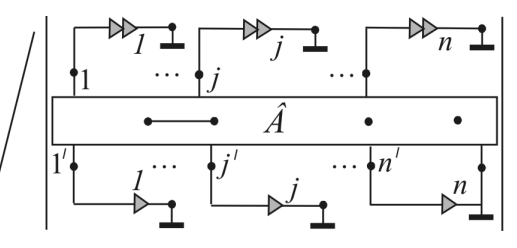

Каждый из элементов $v_{j k}^{\delta}$ и $\mu_{j k}^{\delta}$ является определителем схемы, составленной из двух схем числителей напряжения

Схема числителя в (4) получена из схемы на рис. 1,б путем замены приемника искомого напряжения $\underline{U}_{j}$ нуллатором с номером $n+1$, который управляет многомерным источником, состоящим из источников напряжения и тока с параметрами $\underline{E}, \underline{E}_{V 1}, \underline{E}_{V 2}, \ldots, \underline{E}_{V n}, \underline{J}$. Схема знаменателя сформирована также из схемы на рис. 1,б при нулевых параметрах независимых источников. Формула (4) может быть записана кратко:

$$
\underline{U}_{j}(k)=-\Delta_{j}(k) / \Delta_{E}^{J}
$$

В (5) символом $\Delta_{E}^{J}(k)$ обозначен определитель схемы знаменателя. Нижний и верхний индексы означают здесь и далее замену соответст- полюса $j$ вида (5) или тока вида (6), полученных при экспериментах с номерами $k=1, \ldots, n$ и $k=0$. Если этот элемент - разность числителей напряжения, то нуллатор подключается между полюсами $j$, а САФ принимает вид:

$v_{j k}^{\delta}\left(\right.$ или $\left.\mu_{j k}^{\delta}\right)=$ 
Если же искомый элемент - разность числителей токов, то нуллатор включается параллельно контуру из двух встречно направленных источников тока, каждый из которых управляется током полюса $j$ одной из схем с номерами $k$ и 0 . Аналогично поступают и при определении разности числителей напряжения, если сторона исследуемой подсхемы не содержит общего полюса.

Искомая матрица параметров определяется из (7) с помощью обратной матрицы разностей числителей воздействий:

$$
\Pi=N_{W}^{\delta}\left(N_{X}^{\delta}\right)^{-1}
$$

Достаточными условиями существования решения (8) являются ненулевые значения определителей СКЭ и матрицы разностей числителей воздействий:

$$
\Delta_{E}^{J} \neq 0 ; \operatorname{det}\left(N_{X}^{\delta}\right) \neq 0
$$

САФ неавтономной подсхемы. Для схемы, не содержащей независимых источников, достаточно провести $n$ экспериментов - по числу независимых полюсов (сторон) подсхемы. Из формулы (8) САФ получается как частный случай при нулевых значениях числителей всех воздействий и откликов в нулевом эксперименте: $x_{j 0}=0, w_{j 0}=0$ для всех полюсов $j=1, \ldots, n$.

САФ для параметров неавтономных проходных четырехполюсников. Схема для определения параметров четырехполюсника получается из схемы на рис. 1, $a$ при числе полюсов (сторон) $n=2$. При двух доступных для измерений полюсов (сторон) требуется проведение двух экспериментов с номерами $k=1,2$.

Формула для параметров четырехполюсника следует из (8) при $n=2$ :

$$
\begin{gathered}
\Pi=\begin{array}{|l|l|}
\hline \Pi_{11} & \Pi_{12} \\
\hline \Pi_{12} & \Pi_{22}
\end{array}= \\
=\frac{1}{x_{1}^{1} x_{2}^{2}-x_{2}^{1} x_{1}^{2}} \begin{array}{|l|l|}
w_{1}^{1} x_{2}^{1}-w_{1}^{2} x_{2}^{1} & -w_{1}^{1} x_{1}^{2}+w_{1}^{2} x_{1}^{1} \\
\hline w_{2}^{1} x_{2}^{2}-w_{2}^{2} x_{2}^{1} & -w_{2}^{1} x_{1}^{2}+w_{2}^{2} x_{1}^{1} \\
\hline
\end{array}
\end{gathered}
$$

где $w_{1}^{1}, w_{1}^{2}, w_{2}^{1}, w_{2}^{2}$ и $x_{1}^{1}, x_{1}^{2}, x_{2}^{1}, x_{2}^{2}$ - числители откликов и воздействий соответственно на сторонах четырехполюсника 1 и 2 (указываются в нижнем индексе, как и ранее) в экспериментах 1 и 2 (верхний индекс).

Формула (10) является универсальной для четырехполюсников - из нее можно получить параметры в любой заданной системе координат. Например, формула для $G$-параметров получается из (10) при $x_{1}=N_{U 1} ; x_{2}=N_{I 2} ; w_{1}=N_{I 1} ; w_{2}=N_{U 2}$, где $N_{I 1}, N_{U 2}, N_{U 1}, N_{I 2}$ - определители схем числителей соответствующих токов и напряжений (верхние индексы 1 и 2 не показаны). Если обозначить определители схем числителей напряжений и токов вида (5) и (6) при эксперименте 1 и 2 как $\Delta$ и $\delta$, то искомые формулы приобретают вид:

$$
\begin{gathered}
G_{11}=\frac{\Delta^{1} \delta^{2}-\delta^{1} \Delta^{2}}{D_{G}} ; G_{12}=\frac{\Delta^{1} \delta_{1}-\delta^{1} \Delta_{1}}{D_{G}} ; \\
G_{21}=\frac{-\Delta_{2} \delta^{2}-\delta_{2} \Delta^{2}}{D_{G}} ; G_{22}=\frac{-\Delta_{2} \delta_{1}-\delta_{2} \Delta_{1}}{D_{G}} ; \\
D_{G}=-\Delta_{1} \delta^{2}-\Delta^{2} \delta_{1} .
\end{gathered}
$$

Пример 1. Диагностика трансформатора в гибридных G-nараметрах. Дана схема электроснабжения на рис. 2, $a$ [11]. Действующий комплекс ЭДС источника $\underline{E}=115 \mathrm{\kappa B}$, ток нагрузки $\underline{J}_{6}=1 \mathrm{\kappa A}$, а сопротивления линий $Z_{1}=Z_{2}=10+20 j$ Ом $(j-$ мнимая единица); $\quad Z_{3}=Z_{4}=15+20 j \quad$ Ом; $Z_{5}=Z_{6}=10+20 j$ Ом. Параметры трансформаторов $T_{1}$ ? и $T_{2}$ ? неизвестны. Для измерения напряжений доступны полюсы 3...6. Требуется найти параметры $T_{1}$ ? по программе CIRSYM.

Исследуемый трансформатор $T_{1}$ представим системой $G$-параметров. Для того чтобы найти определители $\Delta$ и $\delta$ из (11), необходимо провести два эксперимента, при этом число сторон подсхем с неизвестными параметрами $n=4$, поэтому напряжения измеряем на всех четырех доступных полюсах 3...6. Эксперимент с номером $k=1$ проведем при наличии в схеме на рис. 2,a одной нагрузки с током $\underline{J}_{6}$, а эксперимент с $k=2-$ на холостом ходу.

Положим, что $G$-параметры обоих трансформаторов следующие: проводимости $G_{11}=G_{33}=-j 1 . e-4 \mathrm{CM}$; сопротивления $G_{22}=G_{44}=0,5+1 j$ Ом; коэффициенты трансформации $G_{21}=G_{43}=0,1$ и $G_{12}=G_{34}=-0,1$, где нумерация параметров соответствует номерам полюсов на рис. 2,a. По программе CIRSYM:

$$
\begin{aligned}
& U_{31}=U_{51}=1,142757582 \cdot 10^{5} e^{-j 0,007735398746} \mathrm{~B} ; \\
& U_{41}=11192,71049 e^{-j 0,05259381308 \mathrm{~B} ;} \\
& U_{61}=12258,56224 e^{-j 1,0426734695} \mathrm{~B} ; \\
& U_{32}=U_{52}=1,147704020 \cdot 10^{5} e^{j 0,0009980036600 \mathrm{~B} ;} \\
& U_{42}=U_{62}=11477,04020 e^{j 0,0009980036600 \mathrm{~B},}
\end{aligned}
$$

где первый индекс означает номер узла схемы, второй - номер эксперимента.

Используя измеренные напряжения, построим СКЭ на рис. 2,б и в для экспериментов с номерами $k=1$ и 2 соответственно. Параметры ЭДС на схемах 


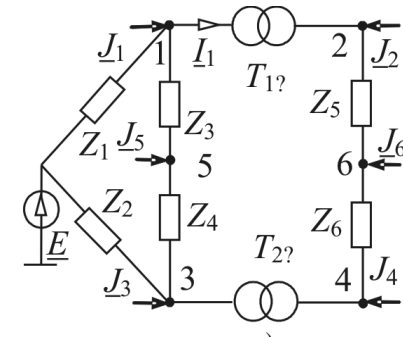

a)

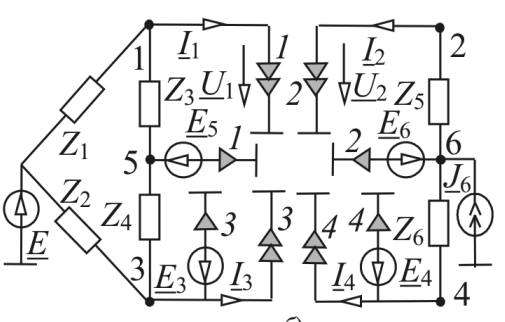

б)

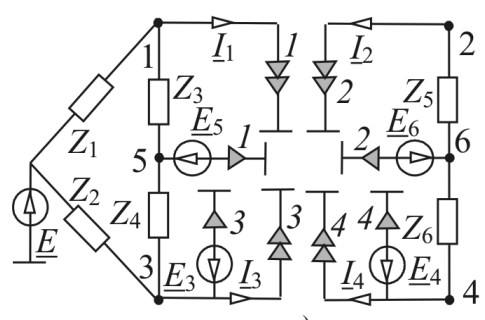

в)

Рис. 2. Схема электроснабжения (a); СКЭ для эксперимента 1 с нагрузочным источником тока $\underline{J}_{6}$ (б) и для эксперимента 2 на холостом ходу (в)

равны напряжениям из (12) согласно нумерации полюсов.

Определители $\Delta^{1}, \Delta_{1}, \Delta^{2}, \Delta_{2}$ из (11) формируются по правилам построения числителей в (5) и (6) с использованием СКЭ на рис. 2,б и раскрываются по программе CIRSYM:

$$
\begin{aligned}
\Delta^{1} & =Z_{2} Z_{6}\left[Z_{4} E+\left(Z_{1}+Z_{3}\right) E_{3}^{1}-\left(Z_{1}+Z_{3}+Z_{4}\right) E_{5}^{1}\right] ; \\
\Delta_{1} & =Z_{1} Z_{2} Z_{6}\left[Z_{3} E_{3}^{1}-\left(Z_{3}+Z_{4}\right) E_{5}^{1}\right] ; \\
\Delta^{2} & =Z_{1} Z_{2} Z_{4}\left[\underline{E}_{4}^{1}-E_{6}^{1}+J_{6} Z_{6}\right] ; \\
\Delta_{2} & =Z_{1} Z_{2} Z_{4}\left[Z_{5} \underline{E}_{4}^{1}-\left(Z_{5}+Z_{6}\right) \underline{E}_{6}^{1}+\underline{J}_{6} Z_{5} Z_{6}\right] \cdot(13)
\end{aligned}
$$

Определители $\delta^{1}, \delta_{1}, \delta^{2}$ и $\delta_{2}$ для (11) формируются из соответствующих определителей (13) путем приравнивания нулю тока $\underline{J}_{6}$ и замены верхнего индекса 1 у символов ЭДС на индекс 2. В результате:

$$
\begin{aligned}
& \delta^{1}=Z_{2} Z_{6}\left[Z_{4} \underline{E}+\left(Z_{1}+Z_{3}\right) E_{3}^{2}-\left(Z_{1}+Z_{3}+Z_{4}\right) E_{5}^{2}\right] \\
& \delta_{1}=Z_{1} Z_{2} Z_{6}\left[Z_{3} E_{3}^{2}-\left(Z_{3}+Z_{4}\right) E_{5}^{2}\right] \\
& \delta^{2}=Z_{1} Z_{2} Z_{4}\left[\underline{E}_{4}^{2}-E_{6}^{2}\right] \\
& \delta_{2}=Z_{1} Z_{2} Z_{4}\left[Z_{5} \underline{E}_{4}^{2}-\left(Z_{5}+Z_{6}\right) E_{6}^{2}\right]
\end{aligned}
$$

Таким образом, символьными выражениями для искомых $G$-параметров трансформатора $T_{1}$ являются формулы (11) с подвыражениями (13) и (14). При подстановке в эти формулы вместо соответствующих ЭДС численных значений напряжений (12) получим $G$-параметры трансформатора с точностью используемой десятичной разрядной сетки.

Пример 2. Диагностика автономной подсхемы. В схеме на рис. 3, $a$ известны проводимости (См) $y_{1}=9, \quad y_{5}=1, y_{8}=1, y_{9}=1, y_{10}=1, \quad y_{11}=1, \quad y_{12}=1$ и $y_{13}=0,5$. Операционный усилитель (ОУ) является идеальным [5]. Для измерения напряжений и токов доступны узлы 4, 5 и 6. Требуется найти неизвестные проводимости $y_{2}$ ? $y_{3}, y_{4}, y_{6}, y_{7}$.
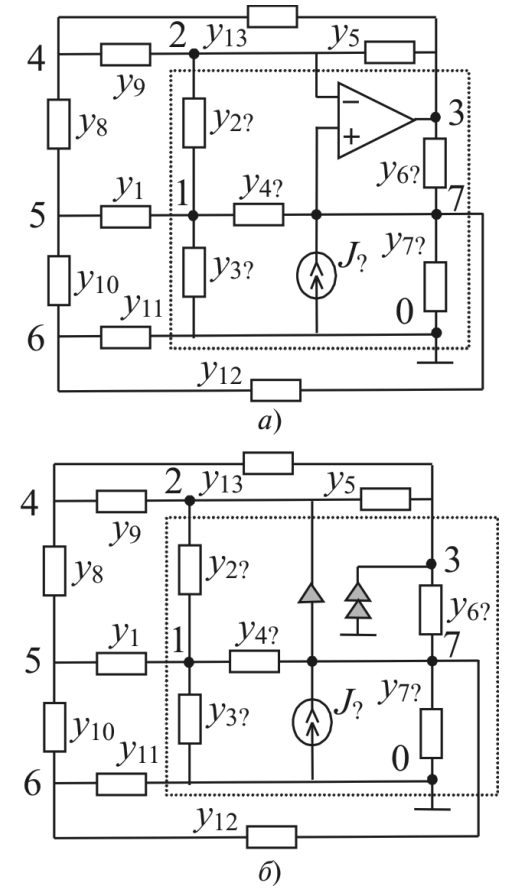

Рис. 3. Исходная схема с автономной подсхемой $(a)$ и эквивалентная схема после замены ОУ нуллором (б)

Искомые проводимости образуют подсхему с $Y$-матрицей размерностью $3 \times 3$, которая получается из матрицы проводимостей схемы без нуллора путем удаления строки, соответствующей полюсу 3, и сложения столбцов, соответствующих полюсам 2 и 7 :

$Y=$\begin{tabular}{|c|c|c|}
\hline$y_{2 ?}+y_{3 ?}+y_{4 ?}$ & $-\left(y_{2 ?}+y_{4 ?}\right)$ & 0 \\
\hline$-y_{2 ?}$ & $y_{2 ?}$ & 0 \\
\hline$-y_{4 ?}$ & $y_{4 ?}+y_{6 ?}+y_{7 ?}$ & $-y_{6 ?}$ \\
\hline
\end{tabular}

По программе CIRSYM проводятся четыре вычислительных эксперимента с номерами $k=0, \ldots, 3$, при этом неизвестные параметры: проводимости (CМ) $y_{2}$ ? $=1 ; y_{3}$ ? $=1 ; y_{4}$ ? $=3 ; y_{6 ?}=0,1 ; y_{7 ?}=1$; ток источника $J_{?}=10$ А [5]. В эксперименте с $k=0$ полюсы с номерами 4, 5 и 6 разомкнуты. В эксперименте с $k=1$ и 2 полюсы 4 и 5 соответственно замыкаются с общим полюсом, а при $k=3$ полюсы 4 и 6 замыкаются между собой. В результате:

$$
U_{4}^{0}=4,8440241345372074
$$


$U_{5}^{0}=3,9796311352646669 ;$

$U_{6}^{0}=2,9997004578715392$;

$I_{40}^{1}=11,848440443793176$;

$U_{5}^{1}=2,5539041239271509$;

$U_{6}^{1}=2,2817667992463889$;

$U_{4}^{2}=2,1140294682895580$;

$I_{50}^{2}=9,9295323510570147$;

$U_{6}^{2}=8,9686098654708520$;

$I_{46}^{3}=3,0787913422387313$;

$U_{5}^{3}=4,4288877770000000$;

$U_{6}^{3}=4,3645974712479463$,

где верхний индекс - номер $k$; нижний индекс: у символа $U-$ номер полюса, на котором измеряется напряжение (В); у символа $I$ - номера замкнутых полюсов, между которыми измеряется ток (А).

Искомая матрица $Y$-параметров определяется по (8). Для этого по напряжениям и токам из (16) строятся четыре СКЭ, соответствующие каждому из четырех экспериментов. По СКЭ и программе CIRSYM можно найти символьное выражение матрицы разностей числителей воздействий (напряжений), которое после подстановки численных значений, преобразуется к виду:

$$
N_{X}^{\delta}=\begin{array}{c|r|r|}
5,06051966 & 14,50678799 & -2,02817636 \\
3,27633285 & 10,47999276 & -2,54989374 \\
-17,02962970 & 4,64821430 & -7,77892484 \\
\hline
\end{array},
$$

где строки и столбцы соответствуют номерам полюсов 1, 2, 7 и номерам экспериментов 1, 2, 3 .

Матрица разностей числителей откликов (токов) определяется также по СКЭ и программе CIRSYM и имеет вид:

$$
N_{W}^{\delta}=\begin{array}{|c|c|c|}
12,19726697 & 30,61396898 & 0,586932115 \mathrm{e}-1 \\
-1,78418682 & -4,026795246 & -0,521717400 \\
-0,45631381 \mathrm{e}-1 & -1,017215136 & -3,592142810 \\
\hline
\end{array}
$$

где строки и столбцы соответствуют тем же полюсам и экспериментам, как и в матрице (17).

Подстановка (17) и (18) в (8) дает матрицу проводимостей

$$
Y=\begin{array}{c|c|c|}
\hline 5,000000048 & -4,000000055 & -0,1448000000 \mathrm{e}-8 \\
-1,000000018 & 1,000000024 & -0,2000000000 \mathrm{e}-9 \\
-3,000000035 & 4,100000040 & -0,99999999882 \mathrm{e}-1 \\
\hline
\end{array}
$$

Искомые значения проводимостей определяются сравнением взаимно соответствующих символьной (15) и численной (19) узловых матриц. При этом точными оказываются 8 десятичных разрядов. Ток источника $J_{\text {? }}$ определяется по программе
CIRSYM и данным однократно проведенного любого из экспериментов [6].

Работа выполнена при поддержке Российского фонда фундаментальных исследований, грант 15-07-05847.

\section{СПИСОК ЛИТЕРАТУРЫ}

1. Киншт Н.В., Герасимова Г.Н., Кац М.А. Диагностика электрических цепей.- М.: Энергоатомиздат, 1983, 192 с.

2. Демирчян К.С., Бутырин П.А. Моделирование и машинный расчет электрических цепей.- М.: Высшая школа, 1988, 335 c.

3. Starzyk J.A. Topological Analysis and Diagnosis of Analog Circuits. - Wydawnictwo PŚ, 2007, 147 p.

4. Al-Naima F.M. and Al-Jewad B.Z. Fault Diagnosis in Analog Circuits via Symbolic Analysis Techniques. - Analog Circuits, InTech, 2013, pp. 91-119.

5. Киншт Н.В., Петрунько Н.Н. Тестовая диагностика активных электрических цепей на основе коммутационных воздействий. - Электричество, 2014, № 6, с. 49-55.

6. Курганов С.А., Филаретов В.В. Формулы для символьной параметрической диагностики линейных электрических цепей. - Электричество, 2006, № 4, с. 47-60.

7. Горшков К.С., Курганов С.А., Филаретов В.В. Диагностика линейных электрических цепей на основе косвенной компенсации подсхем с неизвестными параметрами. - Международный сборник научных трудов «Синтез, анализ и диагностика электронных цепей». - Ульяновск: УГТУ, 2016, с. 92-107.

8. Филаретов В.В. Топологический анализ электронных схем методом выделения параметров. - Электричество, 1998, № 5 , с. $43-52$.

9. Filaretov V.V., Korotkov A.S. Generalized parameter extraction method in case of multiple excitation. - Proc. 8th Int Workshop on Symbolic methods and applications to circuit design (SMACD 04), Wroclaw (Poland), Sept. 2004, pp. 8-11.

10. Филаретов В.В. Программа символьного анализа CIRSYM: история создания, структура и функции. - Международный сборник научных трудов «Синтез, анализ и диагностика электронных цепей». - Ульяновск: УГТУ, 2010, вып.10, с. $158-171$.

11. Хачатрян В.С., Бадалян Н.П., Хачатрян К.В. Метод построения и коррекции узловых сопротивлений при учете комплексных коэффициентов трансформации. - Электричество, 2009, № 8, c. 27-32.

[13.07.17]

А в $m$ о $p$ ы: Гориков Константин Сергеевич окончил в 2006 г. Ульяновский государственный технический университет (УГТУ). В 2010 г. защитил кандидатскую диссертацию «Структурный синтез и символьный допусковый анализ электрических цепей методом схемных определителей». Доцент кафедры электротехники и прецизионных электромеханических систем Санкт-Петербургского национального исследовательского университета информационных технологий, механики и оптики.

Курганов Сергей Александрович окончил в 1978 г. Ульяновский политехнический институт (ныне УГТУ). В 2006 г. защитил докторскую диссертацию «Символьный анализ и диакоптика линейных электрических иепей». Заместитель заведующего кафедрой «Электроснабжение» УГТУ. 
Филаретов Владимир Валентинович окончил в 1982 2. Ульяновский политехнический институт (ныне - УГТУ). В 2002 г. защитил докторскую дис-

Elektrichestvo (Electricity), 2017, No. 12, pp. 36-42 сертацию «Топологический анализ электрических иепей на основе схемного подхода». Профессор кафедры «Электроснабжение» УГТУ.

\section{Diagnostics of Linear Electric Circuits with Indirect Compensation of Subcircuits under the Conditions of a Multiple Experiment}

GORSHKOV Konstantin S. (St. Petersburg National Research University of Information Technologies, Mechanics and Optics, St. Petersburg, Russia) - Associate Professor, Cand. Sci. (Eng.)

KURGANOV Sergei A. (Ul'yanovsk State Technical University (USTU), Ul'yanovsk, Russia) - Professor, Dr. Sci. (Eng.)

FILARETOV Vladimir V. (USTU), Ul'yanovsk, Russia) - Professor, Dr. Sci. (Eng.)

The problem of diagnosing an arbitrary subcircuit within a linear electric circuit is reduced to the problem of analyzing the circuits obtained from the results of a multiple experiment based on the indirect compensation theorem. By using the proposed circuit-and-algebraic formula it becomes possible, unlike the nodal block-matrix formula, to perform diagnostics in an arbitrary coordinate basis and to directly obtain any parameters of the subcircuit under study. The formula can be implemented using well-known algorithms and programs for symbolic or numerical analysis of electric circuits.

K e y w o r d s: electric circuits, diagnostics, multiple experiment, compensation approach, voltage and current clampers

\section{REFERENCES}

1. Kinsht N.V., Gerasimova B.N., Kats M.A. Diagnostika elektricheskikh tsepei (Diagnostics of electrical circuits). Moscow, Energoatomizdat, 1983, 192 p.

2. Demirchyan K.S., Butyrin P.A. Modelirovaniye $i$ mashinnyi raschet elektricheskikh tsepei (Modeling and machine calculation of electrical circuits). Moscow, Publ. «Vysshaya shkola», 1988, 335 p.

3. Starzyk J.A. Topological Analysis and Diagnosis of Analog Circuits. - Wydawnictwo PŚ, 2007, 147 p.

4. Al-Naima F.M. and Al-Jewad B.Z. Fault Diagnosis in Analog Circuits via Symbolic Analysis Techniques. - Analog Circuits, InTech, 2013, pp. 91-119.

5. Kinsht N.V., Petrun'ko N.N. Elektrichestvo - in Russ. (Electricity), No. 6, pp. 49-55.

6. Kurganov S.A., Filaretov V.V. Elektrichestvo - in Russ. (Electricity), 2006, No. 4, pp. 47-60.

7. Gorshkov K.S., Kurganov S.A., Filaretov V.V. Mezhdunarodnyi sbornik nauchnykh trudov «Sintez, analiz $i$ diagnostika elektronnykh tsepei» (International Collection of Scientific Works «Synthesis, Analysis and Diagnostics of Electronic Circuits» ). Ul'yanovsk State Technical University, 2016, pp. 92-107.

8. Filaretov V.V. Elektrichestvo - in Russ. (Electricity), 1998, No. 5, pp. 43-52.

9. Filaretov V.V., Korotkov A.S. Generalized parameter extraction method in case of multiple excitation. - Proc. 8th Int Workshop on Symbolic methods and applications to circuit design (SMACD`04), Wroclaw (Poland), Sept. 2004, pp. 8-11.

10. Filaretov V.V. Mezhdunarodnyi sbornik nauchnykh trudov «Sintez, analiz $i$ diagnostika elektronnykh tsepei» (International Collection of Scientific Works «Synthesis, Analysis and Diagnostics of Electronic Circuits»). Ul'yanovsk State Technical University, 2010, iss. 10, pp. 158-171.

11. Khachatryan V.S., Badalyan N.P., Khachatryan K.V. Elektrichestvo - in Russ. (Electricity), 2009, No. 8, pp. 27-32.

[13.07.17] 